\title{
WAVE PACKET PARAMETRICES FOR EVOLUTIONS GOVERNED BY PDO'S WITH ROUGH SYMBOLS
}

\author{
JEREMY MARZUOLA, JASON METCALFE, AND DANIEL TATARU
}

(Communicated by David S. Tartakoff)

\section{INTRODUCTION}

In this article we consider evolution equations of the form

$$
\left\{\begin{array}{c}
\left(D_{t}+a^{w}(t, x, D)+i b^{w}(t, x, D)\right) u=f, \quad \text { in } \mathbb{R}^{+} \times \mathbb{R}^{n}, \\
u(0)=u_{0}, \quad \text { in } \mathbb{R}^{n},
\end{array}\right.
$$

where $a(t, x, \xi)$ and $b(t, x, \xi)$ are real symbols which are continuous in $t$ and smooth with respect to $x$ and $\xi$.

The operator $a^{w}(t, x, D)$ is selfadjoint; if $b=0$, then this formally guarantees that the above evolution is $L^{2}$ well-posed and the corresponding evolution operators $S(t, s)$ are $L^{2}$ isometries. The $b^{w}$ term rougly contributes to the growth or decay of energy along the flow, depending on whether $b$ is negative or positive.

We are interested in the phase space localization properties of the evolution operators $S(t, s)$. These are best described in terms of the Bargman transform,

$$
(T f)(x, \xi)=2^{-\frac{n}{2}} \pi^{-\frac{3 n}{4}} \int e^{-\frac{1}{2}(x-y)^{2}} e^{i \xi(x-y)} f(y) d y,
$$

which is an isometry from $L^{2}\left(\mathbb{R}^{n}\right)$ to the subspace of $L^{2}\left(\mathbb{R}^{2 n}\right)$ of functions satisfying the Cauchy-Riemann type relation

$$
i \partial_{\xi} T f=\left(\partial_{x}-i \xi\right) T f .
$$

The inversion formula is

$$
f(y)=2^{-\frac{n}{2}} \pi^{-\frac{3 n}{4}} \int e^{-\frac{1}{2}(x-y)^{2}} e^{i \xi(y-x)}(T f)(x, \xi) d x d \xi .
$$

Then one would like to describe the phase space localization of $S(t, s)$ relative to the Hamilton flow corresponding to (1.1). This is given by

$$
\left\{\begin{array}{c}
\dot{x}=a_{\xi}(t, x, \xi) \\
\dot{\xi}=-a_{x}(t, x, \xi) .
\end{array}\right.
$$

Received by the editors November 8, 2006.

2000 Mathematics Subject Classification. Primary 35S10.

The work of the second author was supported in part by an NSF postdoctoral fellowship.

The work of the first and third authors was supported by NSF grants DMS0354539 and DMS0301122. 
We denote by $\chi(t, s)$ the corresponding family of cannonical transformations, and we denote by

the trajectories of the Hamilton flow.

$$
t \rightarrow\left(x^{t}, \xi^{t}\right)
$$

This problem has already been considered in [8], [2]. There one considers the class $S_{00}^{0,(k)}$ of symbols which satisfy the bounds

$$
\left|\partial_{x}^{\alpha} \partial_{\xi}^{\beta} a(x, \xi)\right| \leq c_{\alpha \beta}, \quad|\alpha|+|\beta| \geq k .
$$

The main result has the form

Theorem 1 ([8], [2]). Assume that the symbol $a(t, x, \xi)$ satisfies $a(t, x, \xi) \in S_{00}^{0,(2)}$ uniformly with respect to $t$. Then

(a) The Hamilton flow is bilipschitz.

(b) The kernel $K(t, s)$ of the phase space operator $T^{*} S(t, s) T$ decays rapidly away from the graph of the Hamilton flow,

$$
|K(t, x, \xi, s, y, \eta)| \lesssim(1+|(x, \xi)-\chi(t, s)(y, \eta)|)^{-N} .
$$

However, for applications to nonlinear evolution equations one would like to relax the above class of symbols and replace uniform bounds by an integrability condition. For instance, in the context of the wave equation, related results have been obtained in [7] under assumptions which correspond to replacing the $L^{\infty}$ bounds in (1.6) with $L_{t}^{1} L_{x}^{\infty}$.

In this article we go one step further and restrict the time integrability to the bicharacteristic rays. This is a much more natural condition from the point of view of applications. One motivation for this already appears in early works of Mizohata [5,6] which is concerned with the $b^{w}$ type terms. They consider the equation

$$
L u:=\partial_{t}-i \Delta+\sum_{j=1}^{n} b_{j}(x) \partial_{x_{j}}+c(x, t) u=f(x, t)
$$

and show that a necessary condition for $L$ to be well-posed in $H^{\infty}$ is the bound

$$
\sup _{x \in \mathbb{R}^{n}, \omega \in \mathbb{S}^{n-1}, R>0}\left|\operatorname{Im} \int_{0}^{R} b_{1}(x+r \omega) \cdot \omega d r\right|<\infty .
$$

On the other hand, a slightly stronger version of (1.8) was shown to be sufficient for $L^{2}$ well-posedness in [1].

In the case where $\Delta$ is replaced by the variable coefficient operator $a_{j k}(x, t) \partial_{j} \partial_{k}$, a natural extension of (1.8) is

$$
\sup _{x \in \mathbb{R}^{n},|\xi|=1, R>0}\left|\operatorname{Im} \int_{0}^{R} b_{1}\left(x^{t}(t, x, \xi)\right) \cdot \xi^{t}(t, x, \xi) d r\right|<\infty .
$$

Another motivation for this work comes from the study of general quasilinear Schrödinger equations. In [3] and [4], well-posedness is established in highly regular Sobolev spaces by using estimates for the corresponding linear equation.

Given a symplectic flow $\chi$ in $\mathbb{R} \times \mathbb{R}^{2 n}$, we introduce the symbol class $S^{(k)} L_{\chi}^{1}$ of symbols $q$, which are smooth in $(x, \xi)$, continuous in $t$, and satisfy

$$
\sup _{x, \xi} \int_{0}^{1}\left|\partial_{x}^{\alpha} \partial_{\xi}^{\beta} q(t, \chi(t, 0)(x, \xi))\right| d t \leq c_{\alpha \beta}, \quad|\alpha|+|\beta| \geq k .
$$


Then our condition for the symbol $a$ is implicit; namely $a \in S^{(2)} L_{\chi}^{1}$ where $\chi$ is the Hamilton flow of $a$ defined by (1.5). For the symbol $b$ we will assume that $b \in S^{(1)} L_{\chi}^{1}$. Given such $a$ and $b$, we introduce the notation

$$
\kappa_{N}=\max _{2 \leq|\alpha|+|\beta| \leq N} c_{\alpha \beta}^{a}+\max _{1 \leq|\alpha|+|\beta| \leq N} c_{\alpha \beta}^{b}, \quad \kappa_{0}=\max _{|\alpha|+|\beta|=2} c_{\alpha \beta}^{a}+\max _{|\alpha|+|\beta|=1} c_{\alpha \beta}^{b}
$$

where $c_{\alpha \beta}^{a}$ and $c_{\alpha \beta}^{b}$ are as in (1.9) corresponding to the symbols $a$ and $b$.

The other important parameter in our analysis corresponds to (1.8). We set

$$
M=\sup _{x, \xi} \sup _{0 \leq t_{0} \leq t_{1}} \int_{t_{0}}^{t_{1}} b\left(t, x^{t}, \xi^{t}\right) d t
$$

and assume that $M$ is finite. Then our main result is

Theorem 2. (a) Assume that the symbol $a(t, x, \xi)$ satisfies $a(t, x, \xi) \in S^{(2)} L_{\chi}^{1}$. Then the Hamilton flow defined by (1.5) is globally well defined and bilipschitz.

(b) Assume in addition that $b$ is a symbol in $S^{(1)} L_{\chi}^{1}$ so that $M$ given by (1.11) is finite and the following relation holds for some large $N$ :

$$
e^{2 M} \kappa_{0} \kappa_{4 N} \ll 1 \text {. }
$$

Then the kernel $K(t, s)$ of the phase space operator $T^{*} S(t, s) T$ decays rapidly away from the graph of the Hamilton flow,

$$
|K(t, x, \xi, s, y, \eta)| \lesssim(1+|(x, \xi)-\chi(t, s)(y, \eta)|)^{-N} .
$$

We remark that the smallness in part (b) can be replaced by an equiintegrability condition for the second order derivatives of $a$ along the flow,

$$
\lim _{h \rightarrow 0} \sup _{x, \xi, t_{0}} \int_{t_{0}}^{t_{0}+h}\left|\partial^{2} a\left(x^{t}, \xi^{t}\right)\right| d t=0 .
$$

Then one can take $N$ arbitrary, apply the theorem on sufficiently small time intervals, and iterate.

\section{The Hamilton flow}

In this section we prove that if $a \in S^{(2)} L_{\chi}^{1}$, then the Hamilton flow for (1.1) is well defined and bilipschitz. We first prove the bilipschitz property locally and then use it to show that the flow is globally well defined in the entire time interval.

Thus begin with $\left(x^{0}, \xi^{0}\right) \in \mathbb{R}^{2 n}$. Then there exists some time $t_{0}>0$ and a ball $B$ centered at $\left(x^{0}, \xi^{0}\right)$ so that we can solve $(1.5)$ with initial data $\left(y^{0}, \eta^{0}\right) \in B$. Since, by standard ODE results the flow maps $\chi(t, s)$ are smooth, we only need to obtain the appropriate bounds.

For simplicity, set

$$
\vec{p}(t, x, \xi)=\left(\begin{array}{c}
x^{t}(x, \xi) \\
\xi^{t}(x, \xi)
\end{array}\right)
$$

We have the systems of equations

$$
\begin{aligned}
\partial_{x} \vec{p}(t) & =\left(\begin{array}{l}
1 \\
0
\end{array}\right)+\int_{0}^{t}\left(\begin{array}{cc}
a_{\xi x}(s) & a_{\xi \xi}(s) \\
-a_{x x}(s) & -a_{\xi x}(s)
\end{array}\right) \partial_{x} \vec{p}(s) d s \\
& =\left(\begin{array}{l}
1 \\
0
\end{array}\right)+\int_{0}^{t} A(s) \partial_{x} \vec{p}(s) d s
\end{aligned}
$$

and we have a similar expression for $\partial_{\xi} \vec{p}(t)$ for all $0 \leq t \leq 1$. 
Now, let us see that the flow is Lipschitz in $x$. Taking absolute values and applying Gronwall's inequality, we have

$$
\left\|\partial_{x} \vec{p}(t)\right\| \leq e^{\int_{0}^{t}\|A(s)\| d s} .
$$

However,

$$
\|A\| \lesssim\left|\partial^{2} a(s)\right|
$$

Thus, since $a \in S^{(2)} L_{\chi}^{1}$,

$$
\left\|\partial_{x} \vec{p}(t)\right\| \lesssim 1
$$

and similarly

$$
\left\|\partial_{\xi} \vec{p}(t)\right\| \lesssim 1
$$

Note that bounds on higher derivatives in $x$ and $\xi$ will follow from the same argument.

We have shown that the derivatives of $x^{t}$ and $\xi^{t}$ are uniformly bounded; therefore it is clear that the Hamilton flow map $\chi:(x, \xi) \rightarrow\left(x^{t}, \xi^{t}\right)$ is uniformly bilipschitz in phase space.

It remains to show that the Hamilton flow is globally defined. Consider a bicharacteristic $t \rightarrow\left(x^{t}, \xi^{t}\right)$ starting at $\left(x^{0}, \xi^{0}\right)=(y, \eta)$. This can be continued in time for as long as $\left(x^{t}, \xi^{t}\right)$ remains finite. To prove that this is indeed the case, we consider a one parameter family of bicharacteristics

$$
s \rightarrow\left(x^{t}(s), \xi^{t}(s)\right), \quad\left(x^{s}(s), \xi^{s}(s)\right)=(y, \eta), \quad t \geq s,
$$

and show that $\left(x^{t}(s), \xi^{t}(s)\right)$ is of class $C^{1}$ with respect to $s$ with a uniform Lipschitz bound for $s, t$ in a compact time interval.

Since the Hamilton flow is smooth with a bounded differential, it suffices to estimate the derivative at $s=t$. But this is given by

$$
\frac{d}{d s}\left(x^{t}(s), \xi^{t}(s)\right)_{s=t}=\left(a_{\xi}(t, y, \eta),-a_{x}(t, y, \eta)\right)
$$

and due to the continuity in $t$ it is bounded on any compact time interval.

\section{KERNEL BOUNDS}

We begin with the equation (1.1) for $u$, and, following [8], we derive an equation for its phase space transform $T u$. If the symbol $a$ is linear in $x$ and $\xi$, we have the straightforward identity

$$
T a^{w}(x, D) u=\left(a(x, \xi)+i\left(a_{x} \partial_{\xi}-a_{\xi}\left(\partial_{x}-i \xi\right)\right)\right) T u .
$$

For a general symbol $a$ we denote by $a_{x, \xi}$ its linearization around $(x, \xi)$. Then $\left(T a^{w}(x, D) u\right)(x, \xi)=\left(a(x, \xi)+i\left(a_{x} \partial_{\xi}-a_{\xi}\left(\partial_{x}-i \xi\right)\right)\right) T u(x, \xi)+T\left(a-a_{x, \xi}\right)^{w}(x, D) u$.

This provides us with an evolution equation for $v=T u$,

$$
\left(D_{t}+a+i\left(a_{x} \partial_{\xi}-a_{\xi} \partial_{x}\right)-\xi a_{\xi}+i b\right) v=E v, \quad v(0)=v_{0}=T u_{0},
$$

where the remainder $E$ is given by

$$
E v(x, \xi)=T\left[\left(a-a_{x, \xi}\right)^{w}(x, D)+i\left(b-b_{x, \xi}\right)^{w}(x, D)\right] T^{*} v(x, \xi),
$$


where $b_{x, \xi}$ is the zeroth order term in a Taylor expansion of $b$ about $(x, \xi)$. The kernel $K(t, x, \xi, 0, y, \eta)$ of $T S(t, 0) T^{*}$ is given by the solution $v(t, x, \xi)$ to $(3.1)$ with initial data

$$
\begin{aligned}
v_{0}(x, \xi) & =C_{n} \int_{\mathbb{R}^{n}} e^{i \xi(x-z)} e^{-\frac{(x-z)^{2}}{2}} e^{-i \eta(y-z)} e^{-\frac{(y-z)^{2}}{2}} d z \\
& =C_{n} e^{-\frac{(x-y)^{2}}{4}} e^{-\frac{(\xi-\eta)^{2}}{4}} e^{i \frac{(x-y)(\xi+\eta)}{2}}
\end{aligned}
$$

which decays rapidly away from $(y, \eta)$. Then, the bound (1.12) follows if we show that equation (3.1) is well-posed in weighted $L^{\infty}$ spaces:

Proposition 3.1. Let $a, b$ be as in part (b) of Theorem 2. Then for each initial data $v_{0}$ satisfying

$$
(1+|x-y|+|\xi-\eta|)^{N} v_{0}(x, \xi) \in L^{\infty},
$$

there exists a unique solution $v$ to (3.1) satisfying

$$
\left(1+\left|x-y^{t}\right|+\left|\xi-\eta^{t}\right|\right)^{N} v(t, x, \xi) \in L^{\infty} .
$$

Proof. The inhomogeneous equation

$$
\left(D_{t}+a+i\left(a_{x} \partial_{\xi}-a_{\xi} \partial_{x}\right)-\xi a_{\xi}+i b\right) v=f, \quad v(0)=v_{0},
$$

is an ODE along the Hamilton flow, whose solution satisfies the bound

$$
\begin{aligned}
\left|v\left(t, x^{t}, \xi^{t}\right)\right| & \leq\left|v_{0}(x, \xi)\right| e^{\int_{0}^{t} b\left(\sigma, x^{\sigma}, \xi^{\sigma}\right) d \sigma}+\int_{0}^{t}\left|f\left(s, x^{s}, \xi^{s}\right)\right| e^{\int_{s}^{t} b\left(\sigma, x^{\sigma}, \xi^{\sigma}\right) d \sigma} d s \\
& \leq e^{M}\left(\left|v_{0}(x, \xi)\right|+\int_{0}^{t}\left|f\left(s, x^{s}, \xi^{s}\right)\right| d s\right) .
\end{aligned}
$$

By the bilipschitz property of the flow this implies that

$$
\begin{aligned}
& \left\|\left(1+\left|x-y^{t}\right|+\left|\xi-\eta^{t}\right|\right)^{N} v(t, x, \xi)\right\|_{L^{\infty}} \\
& \lesssim e^{M}\left(\left\|(1+|x-y|+|\xi-\eta|)^{N} v_{0}(x, \xi)\right\|_{L^{\infty}}\right. \\
& \left.\quad+\sup _{x, \xi}(1+|x-y|+|\xi-\eta|)^{N} \int_{0}^{1}\left|f\left(s, x^{s}, \xi^{s}\right)\right| d s\right) .
\end{aligned}
$$

Hence in order to be able to treat the right hand side term $E v$ perturbatively and prove the proposition, it suffices to show that $E$ satisfies the estimate

$$
\begin{aligned}
\sup _{x, \xi}[ & \left.(1+|x-y|+|\xi-\eta|)^{N} \int_{0}^{1}\left|E v\left(s, x^{s}, \xi^{s}\right)\right| d s\right] \\
& \ll e^{-M}\left\|\left(1+\left|x-y^{t}\right|+\left|\xi-\eta^{t}\right|\right)^{N} v(t, x, \xi)\right\|_{L^{\infty}} .
\end{aligned}
$$

For this we have to understand in more detail the kernel of $E$. It suffices to consider the contribution of $a$ in $E$; the estimates for the contribution of $b$ are similar but simpler. We begin with a simple computation for the kernel $K_{q}$ of $T q^{w}(x, D) T^{*}$

$$
\begin{aligned}
& K_{q}\left(x, \xi, x_{1}, \xi_{1}\right) \\
& \quad=C_{n} \int e^{i \xi(x-y)} e^{-\frac{(x-y)^{2}}{2}} e^{i \eta\left(y-y_{1}\right)} q\left(\frac{y+y_{1}}{2}, \eta\right) e^{-i \xi_{1}\left(x_{1}-y_{1}\right)} e^{-\frac{\left(x_{1}-y_{1}\right)^{2}}{2}} d y d y_{1} d \eta .
\end{aligned}
$$

We change variables to

$$
z=\frac{y+y_{1}}{2}, \quad w=\frac{y-y_{1}}{2}
$$


and we integrate with respect to $w$ to obtain

$$
\begin{aligned}
K_{q}(x, & \left.\xi, x_{1}, \xi_{1}\right) \\
& =C_{n} \int e^{i \xi(x-z-w)} e^{-\frac{(x-z-w)^{2}}{2}} e^{2 i \eta w} q(z, \eta) e^{-i \xi_{1}\left(x_{1}-z+w\right)} e^{-\frac{\left(x_{1}-z+w\right)^{2}}{2}} d z d w d \eta \\
& =C_{n} e^{\frac{i\left(x+x_{1}\right)\left(\xi-\xi_{1}\right)}{2}} \int e^{-\frac{\left(\xi+\xi_{1}-2 \eta\right)^{2}}{4}} e^{-\frac{\left(x+x_{1}-2 z\right)^{2}}{4}} q(z, \eta) e^{i \eta\left(x-x_{1}\right)} e^{-i z\left(\xi-\xi_{1}\right)} d z d \eta .
\end{aligned}
$$

Integrating by parts $2 N$ times with respect to $\eta$ and $z$ leads to

$$
\begin{aligned}
& \left|K_{q}\left(x, \xi, x_{1}, \xi_{1}\right)\right| \\
& \quad \lesssim\left(1+\left(x-x_{1}\right)^{2}+\left(\xi-\xi_{1}\right)^{2}\right)^{-N} \\
& \quad \times \int e^{-\frac{\left(\xi+\xi_{1}-2 \eta\right)^{2}}{8}} e^{-\frac{\left(x+x_{1}-2 z\right)^{2}}{8}} \sup _{|\alpha| \leq 2 N}\left|\partial^{\alpha} q(z, \eta)\right| d z d \eta .
\end{aligned}
$$

To estimate this, we use the following:

Lemma 3.2. Let $q$ be a smooth function in $\mathbb{R}^{n}$ with $q(0)=0, \nabla q(0)=0$. Then

$$
\int_{|x| \lesssim R}|q(x)| d x \lesssim R^{n+1} \int_{|x| \lesssim R}|x|^{1-n}\left|\partial^{2} q(x)\right| d x .
$$

Proof. The argument follows directly from the fundamental theorem of calculus. We have

$$
\begin{aligned}
\int_{|x| \lesssim R}|q(x)| d x & \lesssim \int_{0}^{R} \int_{S^{n-1}} \int_{0}^{r} \int_{0}^{r_{1}}\left|\partial_{r}^{2} q\left(r_{2}, \omega\right)\right| d r_{2} d r_{1} r^{n-1} d r d \omega \\
& \lesssim \int_{0}^{R} \int_{S^{n-1}} \int_{0}^{R}\left|\partial_{r}^{2} q\left(r_{2}, \omega\right)\right| r_{2}^{1-n} r_{2}^{n-1} d r_{2} r^{n} d r d \omega \\
& \lesssim R^{n+1} \int_{|x| \lesssim R}|x|^{1-n}\left|\partial^{2} q\right| d x .
\end{aligned}
$$

We apply the lemma to estimate $K=K_{q}$ with $q=a-a_{x, \xi}$ and $N=0$. This gives the crude bound

$$
\left|K\left(x, \xi, x_{1}, \xi_{1}\right)\right| \lesssim\left(1+\left|\xi-\xi_{1}\right|+\left|x-x_{1}\right|\right)^{2 n+3} \int k(x-z, \xi-\eta)\left|\partial^{2} a(z, \eta)\right| d z d \eta
$$

where $k$ is the integrable kernel

$$
k(x, \xi)=(|x|+|\xi|)^{1-2 n}(1+|x|+|\xi|)^{-2} .
$$

Applying the same bound with large $N$ yields

$$
\begin{aligned}
& \left|K\left(x, \xi, x_{1}, \xi_{1}\right)\right| \\
& \lesssim\left(1+\left|\xi-\xi_{1}\right|+\left|x-x_{1}\right|\right)^{2 n+3-4 N} \\
& \quad \times \int k(x-z, \xi-\eta) \sup _{2 \leq|\alpha| \leq 4 N}\left|\partial^{\alpha} a(z, \eta)\right| d z d \eta .
\end{aligned}
$$


Now we can get bounds on $E$ :

$$
\begin{aligned}
& \int_{0}^{1} \mid E v\left(t, x^{t}, \xi^{t}\right) \mid d t \\
& \lesssim \sup _{t \in[0,1]} \int\left(1+\left|x^{t}-x_{1}\right|+\left|\xi^{t}-\xi_{1}\right|\right)^{2 n+3-2 N}\left|v\left(t, x_{1}, \xi_{1}\right)\right| d x_{1} d \xi_{1}, \\
& \int_{0}^{1}\left(\int k\left(x^{t}-z, \xi^{t}-\eta\right)\left|\partial^{2} a(z, \eta)\right| d z d \eta\right)^{\frac{1}{2}} \\
& \quad \times\left(\int k\left(x^{t}-z, \xi^{t}-\eta\right) \sup _{2 \leq|\alpha| \leq 4 N}\left|\partial^{\alpha} a(z, \eta)\right| d z d \eta\right)^{\frac{1}{2}} d t \\
& \lesssim \sup _{t \in[0,1]} \int\left(1+\left|x^{t}-x\right|+\left|\xi^{t}-\xi\right|\right)^{2 n+3-N}|v(t, x, \xi)| d x d \xi \\
& \quad \times\left(\int k\left(x^{t}-z, \xi^{t}-\eta\right)\left|\partial^{2} a(z, \eta)\right| d z d \eta d t\right)^{\frac{1}{2}} \\
& \quad \times\left(\int k\left(x^{t}-z, \xi^{t}-\eta\right) \sup _{2 \leq|\alpha| \leq 4 N}\left|\partial^{\alpha} a(z, \eta)\right| d z d \eta d t\right)^{\frac{1}{2}} .
\end{aligned}
$$

In the last two integrals we change variables $(z, \eta) \rightarrow\left(y^{t}, \zeta^{t}\right)$, which uses the boundedness proved in Section 2. Since $\chi(t, s)$ are bilipschitz, we can replace $k\left(x^{t}-y^{t}, \xi^{t}-\zeta^{t}\right)$ by $k\left(x^{0}-y^{0}, \xi^{0}-\zeta^{0}\right)$. Then we use (1.9) for the time integral and the integrability of $k$ for the $\left(y^{0}, \zeta^{0}\right)$ integral. We obtain

$$
\begin{aligned}
& \int_{0}^{1}\left|E v\left(t, x^{t}, \xi^{t}\right)\right| d t \\
& \quad \lesssim \sqrt{\kappa_{0} \kappa_{4 N}} \sup _{t \in[0,1]} \int\left(1+\left|x^{t}-x\right|+\left|\xi^{t}-\xi\right|\right)^{2 n+3-2 N}|v(t, x, \xi)| d x d \xi
\end{aligned}
$$

where $\kappa_{0}$ and $\kappa_{4 N}$ are as in (1.10).

This allows us to iteratively solve equation (3.1) for initial data $v_{0} \in L^{\infty}$. Indeed, the above estimate implies that

$$
\sup _{x, \xi} \int_{0}^{1}\left|E v\left(t, x^{t}, \xi^{t}\right)\right| d t \lesssim \sqrt{\kappa_{0} \kappa_{4 N}}\|v\|_{L^{\infty}} .
$$

It also allows us to solve equation (3.1) for initial data $v_{0}$ in weighted $L^{\infty}$ spaces. Precisely, for $2 N>4 n+m+3$ we have

$$
\begin{aligned}
\sup _{x, \xi}[ & \left.\left(1+\left|x-x_{1}\right|+\left|\xi-\xi_{1}\right|\right)^{m} \int_{0}^{1}\left|E v\left(t, x^{t}, \xi^{t}\right)\right| d t\right] \\
& \lesssim \sup _{x, \xi}\left[\left(1+\left|x^{t}-x_{1}^{t}\right|+\left|\xi^{t}-\xi_{1}^{t}\right|\right)^{m} \sqrt{\kappa_{0} \kappa_{4 N}}\right. \\
& \left.\quad \times \sup _{t \in[0,1]} \int\left(1+\left|x^{t}-y\right|+\left|\xi^{t}-\eta\right|\right)^{2 n+3-2 N}|v(t, y, \eta)| d y d \eta\right] \\
\lesssim & \sqrt{\kappa_{0} \kappa_{4 N}}\left\|\left(1+\left|x-x_{1}^{t}\right|+\left|\xi-\xi_{1}^{t}\right|\right)^{m} v(t, x, \xi)\right\|_{L_{x, \xi}^{\infty} .}
\end{aligned}
$$

Due to the hypothesis in part (b) of Theorem 2, this implies (3.3) and concludes the proof of the proposition. 


\section{REFERENCES}

[1] I. Bejenaru and D. Tataru, Large data local solutions for the derivative NLS equation, preprint, 2006.

[2] H. Koch and D. Tataru, Dispersive estimates for principally normal pseudodifferential operators, Comm. Pure App. Math. 58 (2005), 217-284. MR2094851 (2005m:35323)

[3] C. Kenig, G. Ponce, L. Vega, The Cauchy problem for quasi-linear Schrödinger equations, Invent. Math. 158 (2004), 343-388. MR2096797 (2005f:35283)

[4] C. Kenig, G. Ponce, C. Rolvung, L. Vega, Variable coefficient Schrödinger flows for ultrahyperbolic operators, Adv. Math. 196 (2005), 373-486. MR2166312 (2006c:35269)

[5] S. Mizohata, Some remarks on the Cauchy problem, J. Math. Kyoto Univ. 1 (1961), 109-127. MR0170112 (30:353)

[6] S. Mizohata, Sur quelques equations du type Schrödinger, Journeés Equations aux dérivées partielles, Saint-Jean de Monts, 1981.

[7] D. Tataru, Strichartz estimates for second order hyperbolic operators with nonsmooth coefficients III, J. Amer. Math. Soc. 15, 2 (2002), 419-442. MR1887639 (2003a:35120)

[8] D. Tataru, Phase space transforms and microlocal analysis, Phase space analysis of partial differential equations. Vol. II. Publ. Cent. Ric. Mat. Ennio Giorgi, Scuola Norm. Sup. Pisa (2004), 505-524. MR2208883

Mathematics Department, University of California, Berkeley, California 94720-3840

E-mail address: marzuola@math.berkeley.edu

Mathematics Department, University of California, Berkeley, California 94720-3840

E-mail address: metcalfe@math.berkeley.edu

Mathematics Department, University of California, Berkeley, California 94720-3840

E-mail address: tataru@math.berkeley.edu 